Draft v1.0, 18/05/2020. This manuscript has not been peer reviewed.

The development of visual search behaviours in immersive virtual reality

\author{
David J Harris ${ }^{1}$, Kyle J Hardcastle ${ }^{2}$, Mark R Wilson ${ }^{1}$, Samuel J Vine ${ }^{1}$
}

1: School of Sport and Health Sciences, University of Exeter, Exeter, UK, EX1 2LU

D.J.Harris@exeter.ac.uk, S.J.Vine@exeter.ac.uk, Mark.Wilson@exeter.ac.uk

2: Counter Terrorism Protective Security Operations, Metropolitan Police Service, Lambeth HQ, London, SE1 7LP

Kyle.J.Hardcastle@met.police.uk

Correspondence concerning this article should be addressed to Dr David Harris, School of Sport and Health Sciences, University of Exeter, St Luke's Campus, Exeter, EX1 2LU. Contact: D.J.Harris@exeter.ac.uk 


\section{The development of visual search behaviours in immersive virtual reality}

1

2 Virtual reality (VR) has clear potential for improving simulation training in many industries.

3 Yet, methods for testing the fidelity, validity and training efficacy of VR environments is, in

4 general, lagging behind their adoption. In this study we examined the effectiveness of VR for

5 training Police room searching procedures, and assessed the corresponding development of

6 perceptual-cognitive expertise through eye-tracking indices of search efficiency. Participants

$7 \quad(n=54)$ were assigned to a VR rule-learning and search training game, a search only training

8 game or a no-practice control group. The VR search training group developed more efficient

9 search behaviours (indexed by saccade size, search rate and gaze entropy) and performed better than controls on a novel transfer test. Efficient gaze behaviours learned during training were not, however, evident during the transfer test. These findings demonstrate how VR can be used to develop perceptual-cognitive skills, but also highlight the challenges of achieving transfer of training.

Keywords; fidelity; Police; Policing; training; validity; VR; 


\section{The development of visual search behaviours in immersive virtual reality}

\section{Introduction}

Virtual reality (VR) has huge potential for revolutionising training in a number of high-pressure and safety critical environments. Of particular interest is the training of perceptual-cognitive and motor skills, such as those needed in sport (Bird, 2019), surgery (Moglia et al., 2016), aviation (Oberhauser \& Dreyer, 2017) or defence and security (Lele, 2013). Ultimately, the success of any form of simulation training is determined by whether practice in the simulation transfers to improvements on the corresponding real-world task (Barnett \& Ceci, 2002; Gray, 2019). While there is wider debate in Psychology about how readily skills learned in one context can be transferred to new tasks (Barnett \& Ceci, 2002; Sala \& Gobet, 2017), the ecological validity of VR (Parsons, 2015) means that the potential for transfer is high. Yet, in order to develop perceptual-cognitive and motor skills in VR, it is necessary to establish that these kinds of skills can actually be developed in VR, as the unusual perceptual characteristics of virtual environments may affect how skills are learned (Harris, Buckingham, et al., 2019a; Harris, Buckingham, Wilson, \& Vine, 2019). Few studies have addressed this issue by assessing changes in perceptual-cognitive abilities during virtual training (Gray, 2019; Tirp, Steingröver, Wattie, Baker, \& Schorer, 2015). In this study we aimed to explore the development of perceptual-cognitive skill and transfer of training in the context of Police room searching.

Effective simulations, for skills training or psychological experimentation purposes, must have the requisite levels of fidelity to create realistic behaviour (Gray, 2019; Harris, Bird, Smart, Wilson, \& Vine, 2020; Slater, 2009). Fidelity describes the extent to which the simulation recreates elements of the real task, such as the visual, auditory or haptic information available. Ultimately, if the perceptual input available to the user is the same as (or functionally indistinguishable from) the real skill, then transfer of training is likely. Subtypes of fidelity include physical (visual and auditory realism), psychological (realistic perceptual and cognitive demands) and affective fidelity (realistic emotional responses). In reviewing the use of VR for training perceptual-cognitive skills - abilities such as attention, anticipation and prediction - Gray (2019) identifies psychological fidelity as particularly relevant for transfer of training. Psychological fidelity is the extent to which the VR environment demands the same perceptual information for controlling actions, elicits the same gaze behaviour and places the same cognitive demands on the user as the real 
49

50

51

52

53

54

55

56

57

58

59

60

61

62

63

64

65

66

67

68

69

70

71

72

73

74

75

76

77

78

79

80

81

environment. Despite evidence that VR training can transfer to real world skills (Michalski et al., 2019) there has been little examination of how perceptual-cognitive skills develop in VR. One exception is a study by Tirp et al., (2015), who found a development of the gaze behaviour quiet eye (Vickers, 2007) to occur during VR dart throwing practice, as well as transfer to improved performance in real dart throwing. If VR training applications cannot create a high degree of psychological fidelity, then transfer of training will be impaired by the lack of perceptual-cognitive skill development (Gray, 2019; Harris, Buckingham, et al., 2019).

In this study we aimed to examine the development of a perceptual-cognitive skill (visual search) and transfer of training in the context of Police room searching. Officers searching a house must identify evidence pertinent to the current investigation as well as be attentive to indicators of other criminal activity. Currently, non-specialist officers are instructed how to recognise indicators of terrorist-related activity using classroom-based exercises. While this approach promotes explicit learning about early indicators of terrorism it does not allow the more active development of procedural knowledge and perceptualcognitive skill that can be achieved through realistic searching practice (Freeman et al., 2014; Trninic, 2018). An encouraging example of the application of VR simulation to Policing is a training device known as AUGGMED (Automated Serious Game Scenario Generator for Mixed Reality Training). AUGGMED allows Police officers and other first responders to practice decision making under pressure during a multi-user VR simulation of a terrorist attack. Comparison between live exercises and AUGGMED training has suggested similar learning benefits between live and VR training (Saunders, Davey, Bayerl, \& Lohrmann, 2019), but the development of perceptual-cognitive skills, like visual search, was not assessed.

The ability to search for a target item amongst other, distracting, stimuli has been extensively studied in lab-based, computerised tests (e.g. see Wolfe, 2010). Typically, these tasks take the form of searching for a simple shape hidden within an array of similar shapes; a letter ' $\mathrm{T}$ ' hidden amongst letter ' $\mathrm{L}$ 's. This work has shown that visual search ability can be trained, as participants improve their speed and accuracy over repeated trials, and that expert searchers (e.g. doctors inspecting a scan for a tumour) show advanced search behaviours (Eckstein, 2011; Savelsbergh, Williams, Kamp, \& Ward, 2002; Wood, Batt, Appelboam, Harris, \& Wilson, 2014). Typically, searchers show changes in eye movements that serve to make their search more efficient, such as making a larger initial saccade after trial onset or 
making fewer (but longer duration) fixations (Najemnik \& Geisler, 2005). There has been limited work that has looked at real-world visual search because of the inherent messiness of real scenes (Torralba, Oliva, Castelhano, \& Henderson, 2006), but the expertise of real world searchers (e.g. airport luggage security) suggests that expertise can be developed. More effective ways to train search skills in an ecologically valid way would be beneficial, not just for Policing, but other settings like airport security or radiology (Biggs, Kramer, \& Mitroff, 2018).

\section{The present study}

For officers to identify early indicators of radicalisation or terrorism during routine house searches they need 1) knowledge of what to look for, and 2) the ability to perform an effective and thorough search. We developed a searching task in VR that aimed to 1) support the development of task-specific knowledge through gamified learning (Kapp, 2012), and 2) allow practice of the perceptual-cognitive skill of scanning and searching a room. As visual search ability can be trained with simple searching tasks (Sireteanu \& Rettenbach, 1995) we developed a realistic but simple search task as a training tool. During the task, the participant is required to search through a number of household items placed on a table, then find and select the target as quickly as possible. We aimed to test whether, through repeated practice, participants showed a development of visual search behaviours, and whether training could transfer to a more complex searching task (moving around and searching a whole room). In addition, we aimed to examine whether a gamified method of acquiring item-specific knowledge (through trial and error) was more beneficial than explicit instruction (akin to existing classroom training).

\section{Hypotheses}

It was predicted that active search practice would lead to a development of visual search skills (changes in eye movement behaviours) as well as positive transfer to a more complex task. Additionally, it was predicted that gamified learning about items to search for would further enhance search performance in the transfer task. Specifically, it was hypothesised that:

$\mathrm{H}_{1}$ : Participants trained on the full training tool (search practice plus gamified learning) will show better performance on both the training task and a novel transfer task, when compared to participants given explicit instruction and generic search practice, who would in turn show better search performance than those given explicit instruction only. 
114

115

116

117

118

119

120

121

122

123

124

125

126

127

128

129

130

131

132

133

134

135

136

137

138

139

140

141

142

$\mathrm{H}_{2}$ : Search training in VR (full training and search training group) will lead to a greater development of visual search skill, measured through eye movement metrics (e.g. search rate, fixation duration, entropy), when compared to controls.

$\mathrm{H}_{3}$ : Visual search skills learned on the simple training task will transfer to the more complex novel search task.

\section{Methods}

\section{Participants}

54 participants ( 33 female, mean age $=22.6$ years, $S D=3.9$ ) were recruited via convenience sampling from an undergraduate population (see Table 1 for demographics). Participants were provided with details of the study and gave written consent before testing began. Ethical approval was obtained from the University Departmental Ethics Committee prior to data collection. The only exclusion criteria were severe vision problems which would prevent participants from completing the task (but spectacles would still be worn within the headset). To ensure homogeneity across the training groups a number of baseline demongraphics were collected (Table 1). In addition to age and gender, participants were asked whether they had used VR before (Yes/No), how frequently they played video games (ranging from 1 [very often] to 5 [never]), and had their vision checked for stereoacuity (Randot test).

Table 1. Demographic information (mean and standard deviation)

\begin{tabular}{r|rrrrr} 
& $\begin{array}{r}\text { Mean } \\
\text { age }\end{array}$ & $\%$ Male & $\begin{array}{r}\text { Previous VR } \\
\text { use (\%) }\end{array}$ & $\begin{array}{r}\text { Video game } \\
\text { experience (max 5) }\end{array}$ & $\begin{array}{r}\text { 3D vision } \\
\text { (max 10) }\end{array}$ \\
\hline & & & & & \\
Training group & $22.4(3.6)$ & 28 & 76 & $3.3(1.5)$ & $5.6(2.6)$ \\
Search group & $23.6(4.7)$ & 44 & 72 & $4.0(1.0)$ & $6.7(1.6)$ \\
Control group & $21.7(2.3)$ & 29 & 73 & $3.9(0.9)$ & $5.9(2.5)$
\end{tabular}

\section{Design}

In line with common practice for evaluating training interventions, we compared the training tool to both an active and a passive control group. The relevant causal contrast (Karlsson \& Bergmark, 2015) in this case is training as usual, which is classroom-based instruction. This type of explicit instruction received by Police officers in the classroom was replicated in the passive control group and the search only group (i.e. active control), and was compared to a more implicit, gamified version training (training group). As a result, a mixed design was used, with group (full training, active control, passive control) as a betweensubject factor and test (pre versus post intervention) as a within-subject factor. Outcome 
RUNNING TITLE: Visual search in virtual reality

143

144

145

146

147

148

149

150

151

152

153

154

155

156

157

158

159

160

161

162

163

164

165

166

167

168

169

170

171

172

173

174

175

measures were performance on the training task and two transfer tasks, plus visual search behaviours.

\section{Tasks and materials}

\section{Virtual reality tasks}

The virtual environment was programmed using the gaming engine Unity (2018.2.10; Unity technologies, CA) and C\#. The VR task was presented using the HTC-Vive (HTC Inc., Taoyuan City, Taiwan), a 6 degrees of freedom VR-system that consists of one headmounted display and two hand-held controllers. The headset was run on an HP EliteDesk PC with an i7 processer and GeForce GTX Titan V graphics card (NVIDIA Inc., Santa Clara, CA). Gaze behaviour in VR was recorded using the built-in Tobii eye tracker in the HTC Vive. The eye tracker uses binocular dark pupil tracking and samples at $120 \mathrm{~Hz}$ across the full $110^{\circ}$ field of view, to an accuracy of $0.5^{\circ}$. Eye tracking data was accessed using the Tobii Pro software development kit and was converted into fixations using the EYEMMV toolbox for MATLAB (Krassanakis, Filippakopoulou, \& Nakos, 2014), which identifies fixations using a spatial dispersion algorithm. Fixation parameters were set to a minimum duration criterion of $100 \mathrm{~ms}$ and spatial dispersion of $1^{\circ}$ of visual angle (Salvucci \& Goldberg, 2000).

\section{Full training task}

The full training task (Fig. 1a) was based on traditional tests of visual search (Eckstein, 2011; Wolfe, 2010) but adapted into a realistic search activity. Participants were placed in a simulation of a small basement style room (see Fig. 1). In the room was a large table on which were between 9 and 14 household items, such as a can of beans, a cup, a mobile phone and a roll of tape. On each iteration of the task (i.e. each trial) the items were randomly selected and spawned at set locations on the table. Participants were required to locate the target item(s) and select them by making a visual fixation on the desired item while simultaneously squeezing the trigger on the Vive controller handle. Participants could select as many items as they wished on each trial. To end each trial the participant was required to select a button on the wall in front of them using the same method. When the trial ended participants were presented with on-screen feedback about the duration of the trial, how many of the selected items were incorrect, and how many targets they missed.

The underlying task of identifying the correct targets was a rule learning game. Five of the 18 total items were correct targets and 13 were distracters, but the identity of these were initially unknown by the participant who had to deduce them through trial and error. On each trial the number of targets to place in the scene was selected pseudo-randomly from 33 
176

177

178

179

180

181

182

183

184

185

186

187

188

189

190

191

192

193

194

195

196

197

198

199

counterbalanced combinations that ensured all possible combinations appeared (so all participants received a similar 'training dose'). Nine of the possible 13 distracters were also randomly spawned on and placed randomly across the set locations.

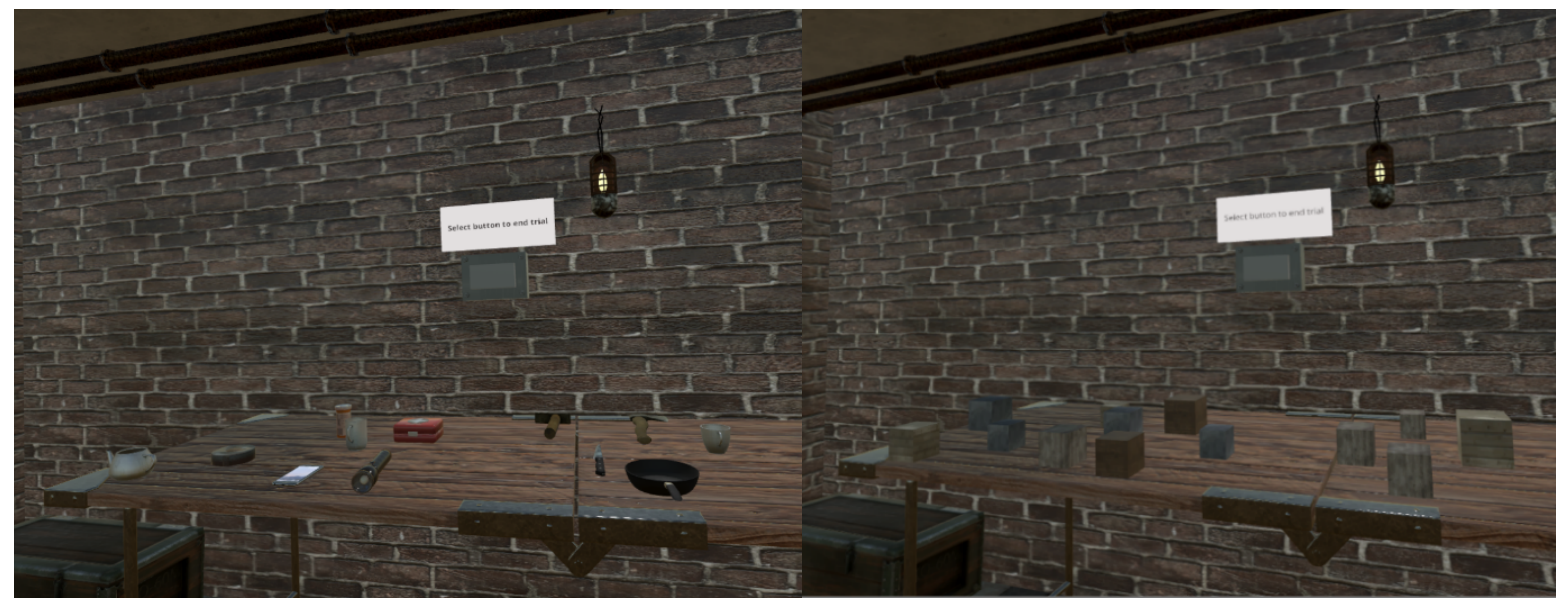

Fig 1a and 1b. The full training task (1a; left panel) and active control (1b; right panel). The participant must search for and select the target items as quickly as possible, then end the trial by selecting the button on the wall.

\section{Search only training task}

The active control task (Fig. 1b) was designed to provide similar searching practice as the full training tool, but without the gamified learning about specific items. Hence the search only task replaced the household items with cubes of three different sizes and three different materials. Participants had to find and select only the metal boxes. This task placed similar demands on visual search, but without the specific item learning and problem-solving demands.

\section{Computerized 2D visual search task}

To test whether the VR search training transferred to improvements on a traditional test of visual search, participants also completed a computerised visual search task (Eckstein, 2011; Wolfe, 2010). This 2D visual search task required participants to identify a target letter ' $\mathrm{T}$ ' in orange colour and regular upright position, presented among other letter ' $\mathrm{T}$ 's of incorrect orientation or colour. Participants were required to press the space bar if the target object appeared, or to withhold if the target object was not present. There were 5, 10, 15, or 20 items in each display array and participants completed 50 arrays (lasting approximately 5 minutes). Performance was indexed through number of mistakes (failure to find the target or 
200

201

202

203

204

205

206

207

208

209

210

211

212

213

214

215

216

217

218

219

220

221

222

failure to withhold the response) and response time. The visual search task was programmed and completed online using psytoolkit.org (Stoet, 2010, 2017).

\section{Transfer task}

The transfer task required a much more active search of a large room, more akin to a real Police house search. The transfer task (Fig. 2) placed the household items at various locations around a room so that the participant had to actively move around the room.

Participants navigated the room using a teleport function. Participants were instructed to be thorough but swift, and chose to end each trial when they had conducted a sufficiently thorough search, as real officers would. Items were not placed in the most obvious locations but were not actively obscured behind other objects. Videos of all three tasks are available online: https://osf.io/5n9j3/.

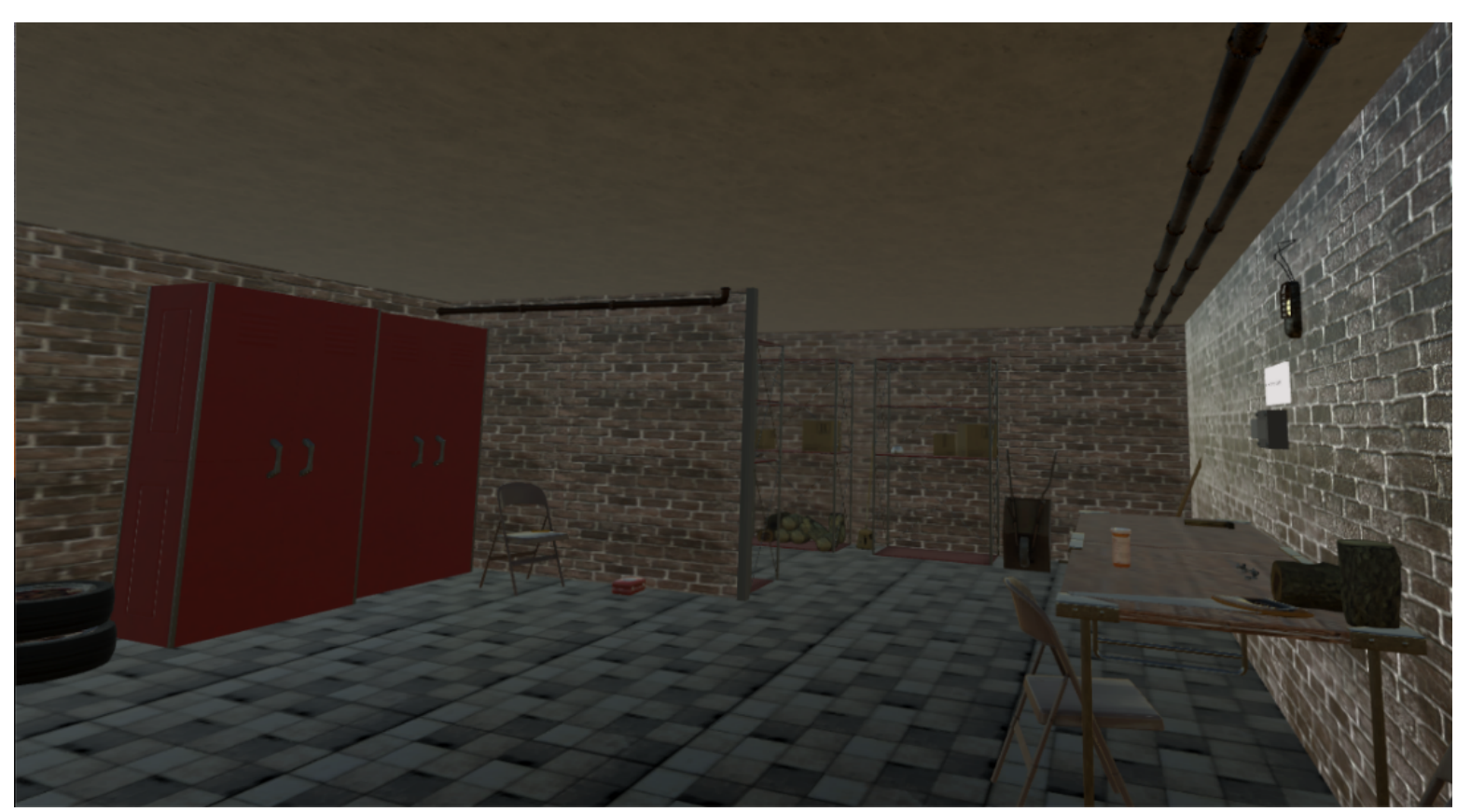

Fig 2. The transfer task. Participants were required to search for items located around the garage.

\section{Measures}

\section{The Simulation Task Load Index (SIM-TLX)}

To assess the level of cognitive demand experienced during the full training task, participants completed the SIM-TLX (Harris, Wilson, \& Vine, 2019). The SIM-TLX is an adaptation of the NASA-TLX (Hart \& Staveland, 1988) for measuring workload during VR simulations. Following completion of the baseline trials on the training task, participants rated the level of demand they had experienced using nine bipolar workload scales: mental 
demands; physical demands; temporal demands; frustration; task complexity; situational stress; distractions; perceptual strain; and task control. For this study we only used the ratings from the 21-point Likert scale, and not the pair-wised comparisons.

\section{Performance}

Performance on the training task, search only training task and transfer test were assessed based on number of errors (combined score of incorrectly selected items and missed items) and the time taken to complete the trial.

\section{Eye tracking metrics}

Saccade size. Previous examinations of visual search have found that participants develop more efficient visual search behaviours as a result of practice. One such improvement is an increase in the size of saccades. Participants learn to more readily identify targets using peripheral vision and become more proficient at making saccades which are larger and land closer to the target (Eckstein, 2011; Mannan, Pambakian, \& Kennard, 2010).

Search rate. Search rate represents the way in which information is extracted from the environment. It is calculated from the number of fixations made, divided by the mean fixation duration (Murray \& Janelle, 2003). Search rate has been identified as a characteristic of expertise in tasks like sport (Mann, Williams, Ward, \& Janelle, 2007; Savelsbergh et al., 2002) and medical image reading (Gegenfurtner et al., 2019). Search behaviour that uses fewer fixations of longer duration is indicative of a knowledge-driven, top-down, less random search strategy (Gegenfurtner et al., 2019). Search rate was calculated relative to time $\left((\text { search rate/time })^{*} 60\right)$ to account for differences in trial duration.

Entropy. In general terms, entropy refers to the uncertainty within a system. When applied to gaze behaviour it can indicate variability or unpredictability of gaze location (Allsop \& Gray, 2014; Moore, Harris, Sharpe, Vine, \& Wilson, 2019; Vine et al., 2015). We adopted a simple measure of entropy (Shannon entropy) to indicate the variability with which gaze was spread across the visual workspace (the visual scene was split into 16 areas of interest and the probability of fixating each was calculated). Shannon entropy (Shannon, 1948) expresses the information contained within a probability distribution in "bits". It is calculated from the state space of the system (all possible outcomes) and the relative probabilities of all elements in that state-space. For instance, if gaze location was random and distributed fairly evenly across all possible locations entropy would be high. If it was targeted to particular locations in a predictable manner entropy would be low. The entropy value was calculated as the sum of the logarithm of all probabilities in the given state space, $H_{(x)}=$ 
$-\sum_{i=1}^{n} P\left(x_{i}\right) \log _{b} P\left(x_{i}\right)$ (as in Shannon, 1948). The probability of fixating each location was calculated for each trial, before applying the above formula to those probabilities.

\section{Procedure}

Participants attended the lab on two occasions lasting up to an hour each. On the first visit they completed the informed consent form and a stereoacuity vision screening test

261 (Stereo Optical Inc., Chicago, IL, United States). Participants then completed the

262 computerised 2D visual search task, followed by a baseline training task assessment (one

263 familiarisation trial plus 10 test trials). Participants were then randomised to one of the three training conditions. Participants in the full training and search only training then completed three blocks of 33 trials on their assigned training. Participants returned for the second visit after a 3-4 day interval. The two training groups then completed a further three blocks of 33 trials on their assigned training (a total of 198 trials). All three groups then completed 10 trials of the training task and repeated the computerised 2D visual search task. Finally, participants completed 10 repetitions on the novel transfer task (see Fig. 3). The active control group and search training group received explicit instruction about what to search for in both the post-test training task the transfer test, but the full training group did not as they

272 had acquired this knowledge through gamified learning. Pilot testing indicated that most

273 participants in the full training group had deduced the correct targets half-way through their 6 274 practice blocks.

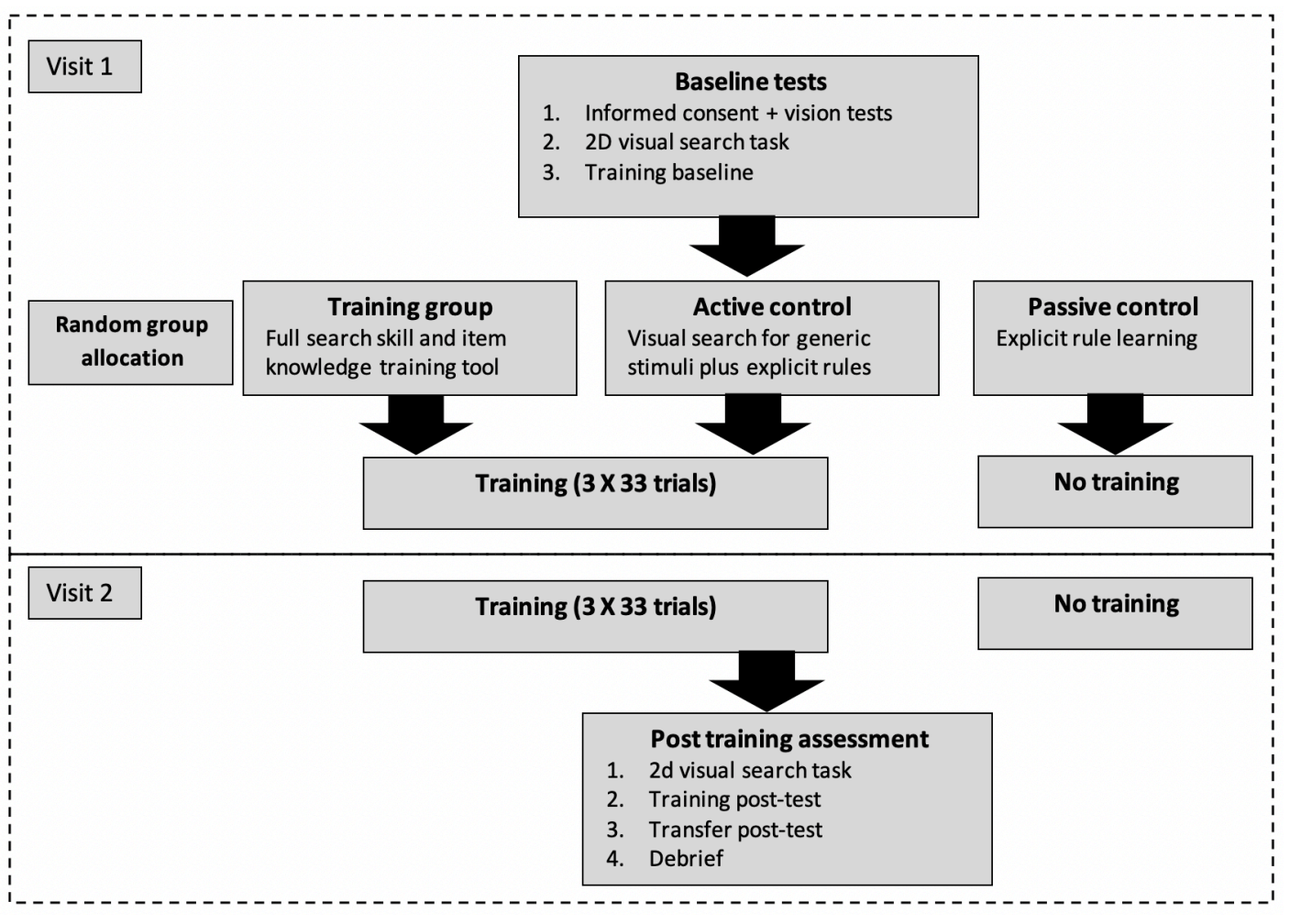


RUNNING TITLE: Visual search in virtual reality

276

277

278

279

280

281

282

283

284

285

286

287

288

289

290

291

292

293

294

295

296

297

298

299

300

301

302

303

304

305

306

307

308

Fig 3. Schematic representation of the flow of participants through the study.

\section{Data analysis}

Data analysis was performed in RStudio v1.0.143 (R Core Team, 2017). Four participants did not return for the second visit (two in the training group and two in the active control group), but were not excluded from the analysis as their baseline data could still be entered into the mixed effects model. Outlying data points (individual trials) more than 3 standard deviations from the mean (Tabachnick \& Fidell, 1996) were Winsorized by replacing the extreme raw score with the next most extreme score (Signorell et al., 2018). Data was also checked for skewness and kurtosis. Completion time for the training task and completion time and errors for the transfer task were found to be positively skewed so a transform was applied for analyses. Linear mixed effects models were run to examine the effect of training group (full training, search only training, control) and trial (pre v post) on performance and eye tracking measures using the lme4 package for R (Bates, Mächler, Bolker, \& Walker, 2014). In order to determine the best fitting model in each instance we initially fitted a near maximal model (Barr, Levy, Scheepers, \& Tily, 2013) - with random effects for group and/or test within the random factor of participant - and then simplified the random effects structure using Principal Components Analysis, as described by Bates, Kliegl, Vasishth, and Baayen (2014). The best fitting model in each instance was chosen by simplifying the structure in line with the number of principal components that contribute to explaining additional variance. Successive models were compared using the Akaike information criterion and likelihood ratio tests. All raw data is freely available from https://osf.io/5n9j3/.

\section{SIM-TLX}

As in previous work Results environment with a descriptive analysis of user's workload. The results (Fig. 4) illustrated that the mental demands and the complexity and frustration arising from the task were moderate $^{1}$. As expected, the physical demands of the task were low. Participants also reported that there was little perceptual strain induced by the simulation and that it was easy to control, suggesting that although it was cognitively effortful, the simulation did not impose additional load as a result of difficulties interacting with it.

\footnotetext{
${ }^{1}$ In the context of the scale maximum of 21.
} 


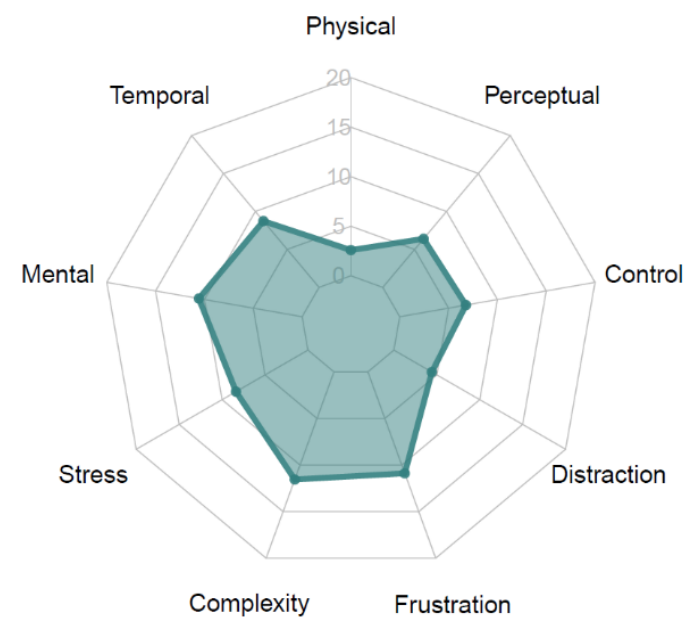

310

311

312

313

314

315

316

317

318

319

320

321

322

323

324

325

326

327

328

329

330
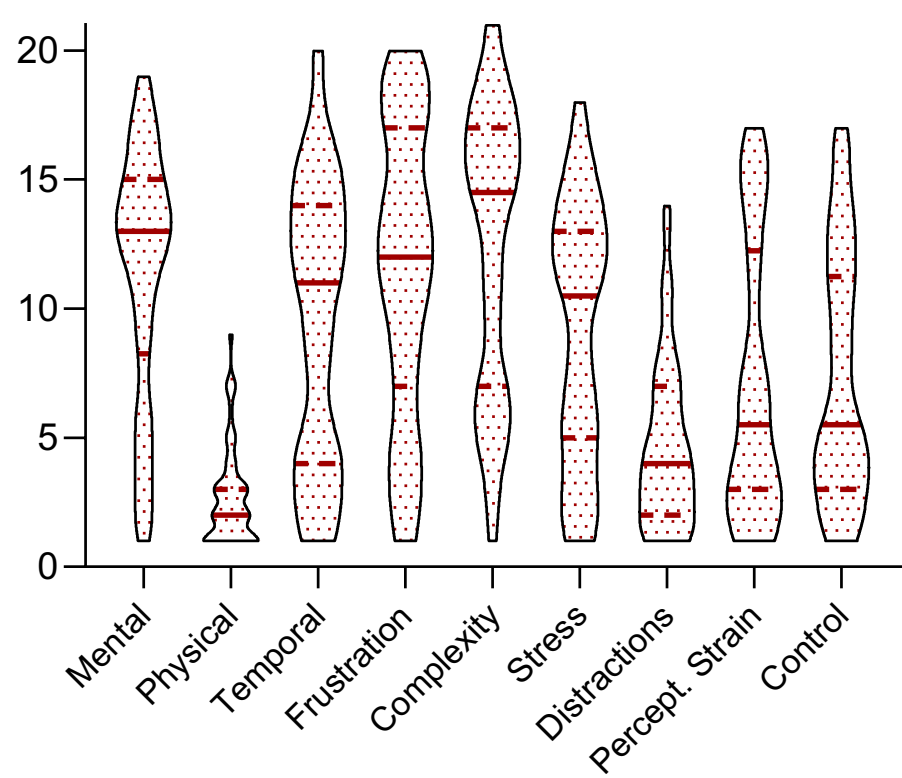

Fig $4 \mathbf{a}$ and $4 \mathbf{b}$. Violin plots with median (solid line) and inter-quartile range (dashed lines) (a; left) and radar chart (b; right) of scores on the SIM-TLX.

\section{Training task}

To assess the effect of training group on training task performance, mixed effects models were run on completion time (seconds) and errors, with participant as a random factor and allowing random effects of test. The overall model predicting time to completion had a total explanatory power (conditional $\mathrm{R}^{2}$ ) of $67.06 \%$, in which the fixed effects explained $45.52 \%$ of the variance. The model's intercept is at 3.15 ( $\mathrm{SE}=0.11,95 \% \mathrm{CI}[2.93,3.37])$. Within this model the effect of group, $F(2,55)=13.73, p<.001, \mathrm{n}_{\mathrm{p}}{ }^{2}=.025$, and the effect of test (pre v post), $F(1,52)=170.11, p<.001, \mathrm{n}_{\mathrm{p}}{ }^{2}=.156$, were both significant. The interaction effect was also significant, $F(2,53)=5.54, p=.007, \mathrm{n}_{\mathrm{p}}{ }^{2}=.010$. Pairwise tests indicated that there were no group differences at baseline ( $\mathrm{ps}>.18)$. At post-test, both the full training group $(p<.001)$ and the search training group ( $p=.01$ ) outperformed the control group. The full training group also searched more quickly than the search only group ( $p=.01$ ) (see Fig 5A).

The overall model predicting errors had a total explanatory power of $84.17 \%$, in which the fixed effects explain $68.27 \%$ of the variance. The model's intercept is at 0.49 ( $\mathrm{SE}=$ $0.12,95 \%$ CI $[0.26,0.72])$. Within this model, the effect of test was significant, $F(1,51)=365.46, p<.001, \mathrm{n}_{\mathrm{p}}{ }^{2}=.293$. However, the effect of group, $F(2,52)=1.66, p=.20$, $\mathrm{n}_{\mathrm{p}}{ }^{2}=.004$ and the interaction effect, $F(2,54)=1.29, p=.28, \mathrm{n}_{\mathrm{p}}{ }^{2}=.003$, were not significant. 


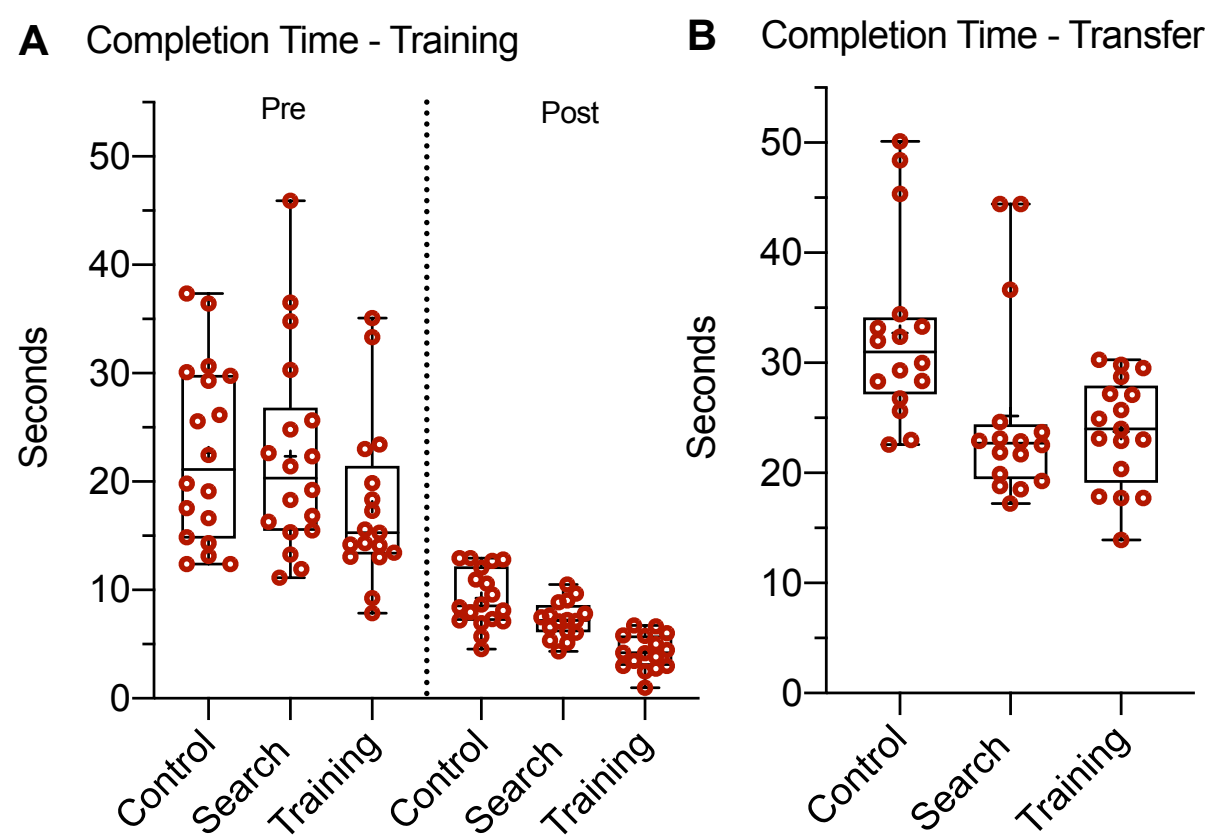

331

332

333

334

335

336

337

338

339

340

341

342

343

344

345

346

347

348

349

350

351

352

Fig 5a and 5b. Box and whisker plot (with data points) showing performance (completion time) on the training task (a; left) and novel transfer task (b; right).

\section{Computerized transfer test}

To test for transfer of training to the 2D visual search task, mixed effects models were run on search time (seconds) and number of errors made, with participant as a random factor. The overall model predicting search rate had a total explanatory power (conditional $\mathrm{R}^{2}$ ) of $51.62 \%$, in which the fixed effects explain $12.47 \%$ of the variance. The model's intercept is at $928.36(\mathrm{SE}=40.66,95 \% \mathrm{CI}[850.27,1006.94])$. Within this model the effect of group, $F(2,47)=3.75, p=.03, \mathrm{n}_{\mathrm{p}}^{2}=.101$, and the effect of test, $F(1,45)=6.91, p=.01, \mathrm{n}_{\mathrm{p}}^{2}=.093$ are both significant. The interaction was not significant, $F(2,45)=0.04, p=.96, \mathrm{n}_{\mathrm{p}}{ }^{2}=.001$. Pairwise tests indicated no significant improvement in any of the three groups, although there was a trend towards an improvement in the training group $(p=.07)$, and the search only group. $(p=.08)$, but not the control group ( $p=.41)$ (see Fig 6).

The overall model predicting search errors had a total explanatory power (conditional $\mathrm{R}^{2}$ ) of $61.91 \%$, in which the fixed effects explain $2.55 \%$ of the variance. The model's intercept is at 0.83 ( $\mathrm{SE}=0.22,95 \% \mathrm{CI}[0.41,1.24])$. Within this model, the effect of group was not significant $F(2,51)=0.22, p=.81, \mathrm{n}_{\mathrm{p}}{ }^{2}=.007$, the effect of test was not significant, $F(1,49)=0.02, p=.90, \mathrm{n}_{\mathrm{p}}{ }^{2}=.000$, and the interaction was not significant, $F(2,49)=2.49, p=.09$, $\mathrm{n}_{\mathrm{p}}{ }^{2}=.076$. 
353

354

355

356

357

358

359

360

361

362

363

364

365

366

367

368

369

370

371

372

373

374

375

376

377

378

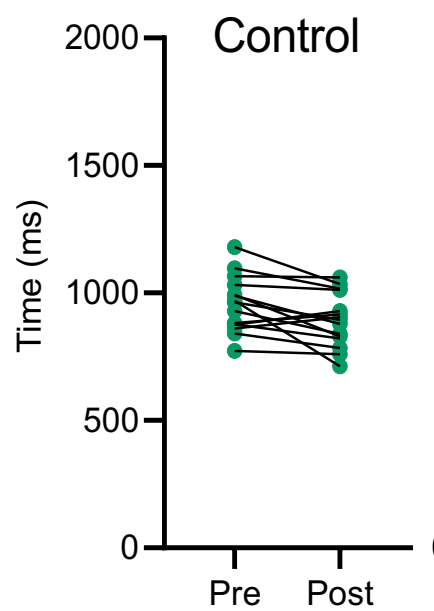

Search

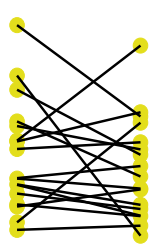

Full
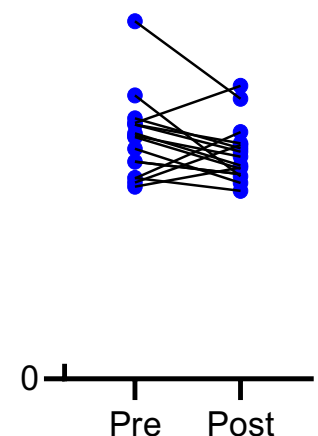

Fig 6. Pre and post raw scores (search time) on computerised visual search task.

\section{Novel transfer test}

To compare effect of training group on performance (time and errors) on the novel transfer test, mixed effects models with participant and trial as random factors were run. The overall model predicting completion time had a total explanatory power of $71.44 \%$, in which the fixed effects explain $11.97 \%$ of the variance. The model's intercept is at 4.89 ( $\mathrm{SE}=0.11$, $95 \%$ CI $[4.67,5.11])$. Within this model the effect of group was significant, $F(2,44)=4.96$, $p=.01, \mathrm{n}_{\mathrm{p}}{ }^{2}=.026$. Bonferroni-holm corrected comparisons indicated that there was no difference between full training and search training groups $(p=.27)$. The search group were significantly better than controls $(p=.01)$ and the difference between full training and controls was marginal ( $p=.058)$ (see Fig 5B).

The overall model predicting errors had total explanatory power of $52.60 \%$, in which the fixed effects explain $0.66 \%$ of the variance. The model's intercept is at 0.39 ( $\mathrm{SE}=0.13$, $95 \%$ CI $[0.14,0.63])$. Within this model, the effect of group was not significant, $F(2,44)=0.27, p=.76, \mathrm{n}_{\mathrm{p}}{ }^{2}=.001$.

\section{Gaze parameters}

Training. To examine changes in gaze behaviour across training mixed effects models were run on saccade size, mean fixation duration and gaze entropy. The overall model predicting saccade size had a total explanatory power of $15.38 \%$, in which the fixed effects explain $5.43 \%$ of the variance (marginal R2). The model's intercept is at 0.23 ( $\mathrm{SE}=$ $0.017,95 \%$ CI $[0.19,0.26])$. Within this model the effect of group was not significant, $F(2,54)=1.75, p=.18, \mathrm{n}_{\mathrm{p}}{ }^{2}=.004$. However, the effect of test, $F(1,49)=26.07, p<.001, \mathrm{n}_{\mathrm{p}}{ }^{2}=.027$ and the interaction, $F(2,50)=4.71, p=.01, \mathrm{n}_{\mathrm{p}}{ }^{2}=.010$ were both significant. Pairwise 
379

380

381

382

383

384

385

386

387

388

389

390

391

392

393

394

395

396

397

398

399

400

401

402

403

404

405

comparisons showed that there were significant increases in saccade size in the training $(p=.01)$ and search $(p<.001)$ groups, but not controls $(p=.43)$.

The overall model predicting search rate had a total explanatory power of $43.13 \%$, in which the fixed effects explain $21.80 \%$ of the variance. The model's intercept is at 0.85 ( $\mathrm{SE}=$ $0.046,95 \%$ CI $[0.76,0.94])$. Within this model the effect of test was significant, $F(1,50)=100.28, p<.001, \mathrm{n}_{\mathrm{p}}{ }^{2}=.101$, but the main effect of group, $F(2,58)=1.33, p=.27$, $\mathrm{n}_{\mathrm{p}}{ }^{2}=.003$ and the interaction were not significant, $F(2,52)=2.00, p=.15, \mathrm{n}_{\mathrm{p}}^{2}=.004$.

The overall model predicting entropy has a total explanatory power of $55.20 \%$, in which the fixed effects explain $7.94 \%$ of the variance. The model's intercept is at 1.94 (SE = $0.085,95 \%$ CI $[1.78,2.11])$. Within this model the effect of group was not significant, $F(2,56)=2.19, p=.122, \mathrm{n}_{\mathrm{p}}{ }^{2}=.005$, but the effect of test, $F(1,49)=6.19, p=.02, \mathrm{n}_{\mathrm{p}}{ }^{2}=.007$, and the interaction, $F(2,51)=4.75, p=.01, \mathrm{n}_{\mathrm{p}}^{2}=.011$, were both significant. Pairwise contrasts indicated no change in entropy in the control $(p=.40)$ or training groups $(p=.50)$, but a significant reduction in the search training group $(p=.001) .^{2}$

Transfer. To compare the gaze behaviour of the training groups during the novel transfer test a mixed effects model was run. The overall model predicting saccade size had a total explanatory power of $13.71 \%$, in which the fixed effects explain $0.33 \%$ of the variance (marginal R2). The model's intercept is at 0.44 ( $\mathrm{SE}=0.053,95 \% \mathrm{CI}[0.33,0.54])$. Within this model the effect of group was not significant, $F(2,48)=0.44, p=.65, \mathrm{n}_{\mathrm{p}}{ }^{2}=.011$.

The overall model predicting search rate had a total explanatory power of $50.81 \%$, in which the fixed effects explain $4.33 \%$ of the variance. The model's intercept is at $0.55(\mathrm{SE}=$ $0.031,95 \%$ CI $[0.49,0.61])$. Within this model the effect of group was not significant, $F(2,44)=1.95, p=.15, \mathrm{n}_{\mathrm{p}}{ }^{2}=.010$.

The overall model predicting entropy had a total explanatory power of $35.93 \%$, in which the fixed effects explain $1.55 \%$ of the variance. The model's intercept is at 2.42 ( $\mathrm{SE}=$ $0.050,95 \%$ CI $[2.32,2.52])$. Within this model the effect of group was not significant, $F(2,48)=0.95, p=.39$.

\footnotetext{
${ }^{2}$ Saccade size, search rate and entropy were all significant predictors of completion time during training $(p s<.02)$, and entropy and search rate were significant predictors of completion time in the transfer test $(\mathrm{ps}<.001)$.
} 

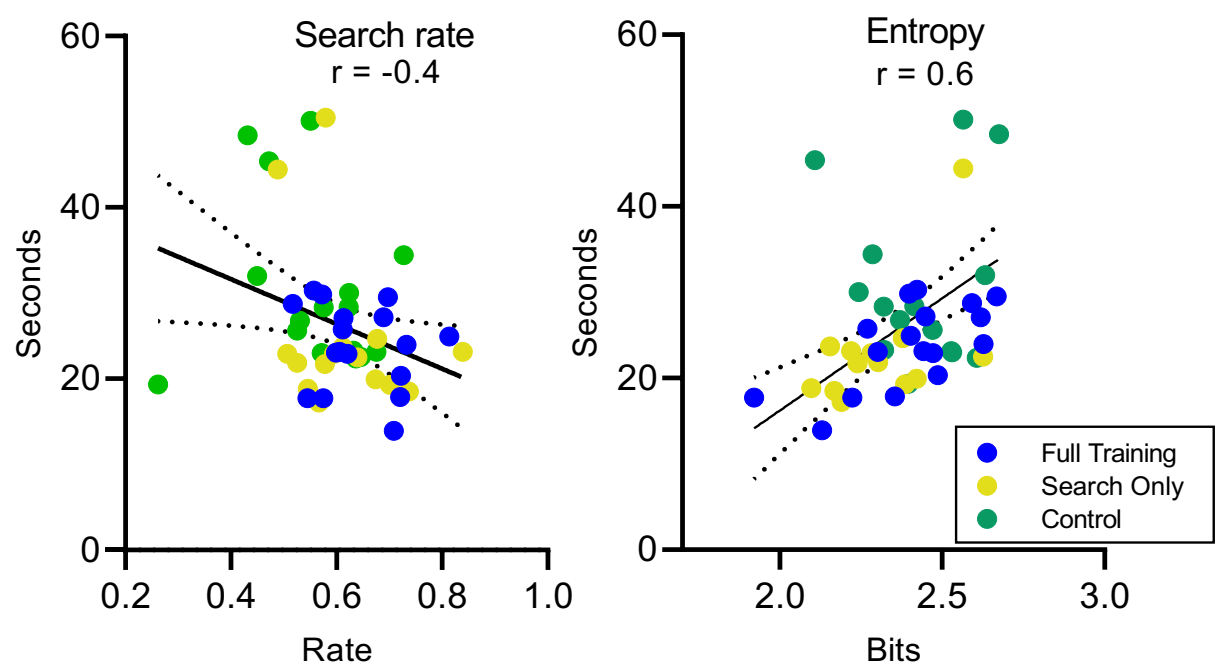

406

407

408

409

410

411

412

413

414

415

416

417

418

419

420

421

422

423

424

425

426

427

428

429

430

Fig $7 \mathbf{a}$ and $7 \mathbf{b}$. Correlations of search rate (a; left) and entropy (b; right) with time to completion in novel transfer test

\section{Discussion}

The increasing accessibility, and decreasing cost, of commercial VR hardware and software has led to considerable interest in VR as training tool, but the effect of VR training on perceptual-cognitive skills is not well understood. In this study we aimed to test the effectiveness of a VR visual search training device, to explore its potential for training Police house searching. We aimed to establish whether perceptual-cognitive expertise for room searching could be developed in VR, and whether training transferred to a more complex search task. These findings are relevant for developing a better understanding of perceptualcognitive skill learning in VR, as well as for practical applications of VR.

Our primary prediction, that active search practice would lead to improved performance on the transfer task, was largely supported, but there was no benefit for the full training group over search only training. The search only training group significantly outperformed controls in the novel transfer test $(p=.01)$, but the difference between controls and full training was only marginal $(p=.058)$. This finding suggests that while active search practice transferred to the novel task, there was no additional benefit from gamified learning about specific items. By contrast, there was no difference between groups in terms of errors committed. As Fig 6 panel D illustrates, participants made few errors in the transfer task (all groups averaged less than 0.5 errors per trial) suggesting that there was a floor effect in this measure. In real-world policing, conducting a search that is both thorough and fast is 
431

432

433

434

435

436

437

438

439

440

441

442

443

444

445

446

447

448

449

450

451

452

453

454

455

456

457

458

459

460

461

462

463

464

important, but the transfer task may not have been sufficiently challenging for us to observe a difference in errors as well as completion time.

As predicted, active search practice (both full and search only training groups) led to changes in gaze behaviour indicative of more efficient search. Following training, all groups showed a decrease in search rate, but only the two active searching groups showed an increase in saccade length and only the search only group showed a decrease in entropy. Few studies have investigated the development of perceptual-cognitive skill in VR, and these findings suggest that, despite potential differences between real and VR environments, visual search behaviours can be learned in VR. This suggests a good degree of psychological fidelity in the VR environment (Harris et al., 2020).

Despite group differences in performance on the transfer task, accompanying differences in gaze behaviour were not evident, so our final hypothesis was not supported. Additionally, there were no group differences present in the computerised visual search task either, suggesting that no generalised improvement in visual search ability occurred. This finding illustrates the challenge of transferring perceptual-cognitive skills to new tasks. Specificity is an important feature of expertise, and the differences between the training and transfer tasks may have prevented any transfer of gaze behaviours (Barnett \& Ceci, 2002; Sala \& Gobet, 2017). In this instance we assessed transfer from a relatively static searching task to one that required locomotion. In the future, the VR training task may have to be more closely aligned with the target task for transfer of gaze behaviours to occur. The benefit of VR training, however, is that tasks can be designed to closely match the real-world scenario.

\section{Limitations}

One limitation of this study is that transfer of training was only assessed in another VR task. The fact that the simple task transferred to a full room search is a promising finding, but transfer of training outside of the virtual world will pose more of a challenge (Barnett \& Ceci, 2002). Nonetheless, the primary aim of this study was to demonstrate the potential for developing search skills in VR, which was supported by the eye movement metrics. Further work should see to establish whether the training will transfer to a real-world scene, and if developments in search behaviour can be transferred when the tasks are more closely aligned.

\section{Conclusions}

If VR is to fulfil its promise and become a productive training methodology in environments like sport, rehabilitation, and defence and security, environments with high levels of psychological fidelity are required. That is, VR training environments must be capable of developing the cognitive and visuomotor abilities that underpin performance in 
RUNNING TITLE: Visual search in virtual reality

465 these domains. In this study we demonstrated that gaze behaviours indicative of visual search 466 expertise (search rate, saccade size and entropy) could be trained in a virtual environment, 467 but that transfer of these skills to new tasks remains a challenge; even in immersive VR.

468 Nonetheless transfer of training to a more complex and ecologically valid task was achieved. 469 The enhanced realism and immersion provided by VR (Parsons, 2015; Slater, 2009), aligned 470 to the motivational (Allcoat \& Mühlenen, 2018; Salar, Arici, Caliklar, \& Yilmaz, 2020) and 471 practical benefits, means that VR is set to play a major role in human skills training.

472 Continued testing of psychological fidelity and transfer of training is needed to ensure that 473 adoption of VR training is evidence based.

474

475

476

477

478

479

480

481

482

483

484

485

486

487

488

489

490

491

492

493

494

495

496

497 
RUNNING TITLE: Visual search in virtual reality

498

499

500

501

502

503

504

505

506

507

508

509

510

511

512

513

514

515

516

517

518

519

520

521

522

523

524

525

526

527

\section{References}

Allcoat, D., \& Mühlenen, A. von. (2018). Learning in virtual reality: Effects on performance, emotion and engagement. Research in Learning Technology, 26(0). Retrieved December 19, 2018, from https://journal.alt.ac.uk/index.php/rlt/article/view/2140

Allsop, J., \& Gray, R. (2014). Flying under pressure: Effects of anxiety on attention and gaze behavior in aviation. Journal of Applied Research in Memory and Cognition, 3(2), $63-71$.

Barnett, S. M., \& Ceci, S. J. (2002). When and where do we apply what we learn?: A taxonomy for far transfer. Psychological Bulletin, 128(4), 612-637.

Barr, D. J., Levy, R., Scheepers, C., \& Tily, H. J. (2013). Random effects structure for confirmatory hypothesis testing: Keep it maximal. Journal of Memory and Language, $68(3), 255-278$.

Bates, D., Mächler, M., Bolker, B., \& Walker, S. (2014). Fitting linear mixed-effects models using lme4. ArXiv:1406.5823 [stat]. Retrieved April 29, 2018, from http://arxiv.org/abs/1406.5823

Biggs, A. T., Kramer, M. R., \& Mitroff, S. R. (2018). Using Cognitive Psychology Research to Inform Professional Visual Search Operations. Journal of Applied Research in Memory and Cognition, 7(2), 189-198.

Bird, J. M. (2019). The use of virtual reality head-mounted displays within applied sport psychology. Journal of Sport Psychology in Action, 0(0), 1-14.

Eckstein, M. P. (2011). Visual search: A retrospective. Journal of Vision, 11(5), 14-14.

Freeman, S., Eddy, S. L., McDonough, M., Smith, M. K., Okoroafor, N., Jordt, H., \& Wenderoth, M. P. (2014). Active learning increases student performance in science, engineering, and mathematics. Proceedings of the National Academy of Sciences, 111(23), 8410-8415.

Gegenfurtner, A., Boucheix, J. M., Gruber, H., Hauser, F., Lehtinen, E., \& Lowe, R. K. (2019). The Gaze Relational Index as a Measure of Visual Expertise. Journal of Expertise, 3(1).

Gray, R. (2019). Virtual environments and their role in developing perceptual-cognitive skills in sports. A.M. Williams \& R.C. Jackson (Eds.) Anticipation and Decision Making in 
RUNNING TITLE: Visual search in virtual reality

528

529

530

531

532

533

534

535

536

537

538

539

540

541

542

543

544

545

546

547

548

549

550

551

552

553

554

555

556

557

Sport. Taylor \& Francis, Routledge. Retrieved from https://doi.org/10.4324/9781315146270-19

Harris, D., Buckingham, G., Wilson, M., Brookes, J., Mushtaq, F., Mon-Williams, M., \& Vine, S. J. (2019a). Testing the effects of virtual reality on visuomotor skills (preprint). PsyArXiv. Retrieved May 5, 2020, from https://osf.io/yke6c

Harris, D., Buckingham, G., Wilson, M., Brookes, J., Mushtaq, F., Mon-Williams, M., \& Vine, S. J. (2019b). Testing the fidelity and validity of a virtual reality golf putting simulator (preprint). PsyArXiv. Retrieved July 15, 2019, from https://osf.io/j2txe

Harris, D. J., Bird, J. M., Smart, A. P., Wilson, M. R., \& Vine, S. J. (2020). A framework for the testing and validation of simulated environments in experimentation and training. Frontiers in Psychology, 11(605).

Harris, D. J., Buckingham, G., Wilson, M. R., \& Vine, S. J. (2019). Virtually the same? How impaired sensory information in virtual reality may disrupt vision for action. Experimental Brain Research, 237(11), 2761-2766.

Harris, D. J., Wilson, M. R., \& Vine, S. J. (2019). Development and validation of a simulation workload measure: The Simulation Task Load Index (SIM-TLX). Virtual Reality.

Hart, S. G., \& Staveland, L. E. (1988). Development of NASA-TLX (Task Load Index): Results of empirical and theoretical research. Advances in Psychology (Vol. 52, pp. 139-183). Elsevier. Retrieved September 3, 2018, from http://linkinghub.elsevier.com/retrieve/pii/S0166411508623869

Kapp, K. M. (2012). The Gamification of Learning and Instruction: Game-based Methods and Strategies for Training and Education. John Wiley \& Sons.

Karlsson, P., \& Bergmark, A. (2015). Compared with what? An analysis of control-group types in Cochrane and Campbell reviews of psychosocial treatment efficacy with substance use disorders. Addiction (Abingdon, England), 110(3), 420-428.

Krassanakis, V., Filippakopoulou, V., \& Nakos, B. (2014). EyeMMV toolbox: An eye movement post-analysis tool based on a two-step spatial dispersion threshold for fixation identification. Journal of Eye Movement Research, 7(1). Retrieved December 21, 2018, from https://bop.unibe.ch/JEMR/article/view/2370 
RUNNING TITLE: Visual search in virtual reality

558

559

560

561

562

563

564

565

566

567

568

569

570

571

572

573

574

575

576

577

578

579

580

581

582

583

584

585

586

587

Lele, A. (2013). Virtual reality and its military utility. Journal of Ambient Intelligence and Humanized Computing, 4(1), 17-26.

Mann, Williams, A. M., Ward, P., \& Janelle, C. M. (2007). Perceptual-Cognitive Expertise in Sport: A Meta-Analysis. Journal of Sport and Exercise Psychology, 29(4), 457-478.

Mannan, S. K., Pambakian, A. L., \& Kennard, C. (2010). Compensatory strategies following visual search training in patients with homonymous hemianopia: An eye movement study. Journal of neurology, 257(11), 1812-1821.

Michalski, S. C., Szpak, A., Saredakis, D., Ross, T. J., Billinghurst, M., \& Loetscher, T. (2019). Getting your game on: Using virtual reality to improve real table tennis skills. PLoS ONE, 14(9):e0222351.

Moglia, A., Ferrari, V., Morelli, L., Ferrari, M., Mosca, F., \& Cuschieri, A. (2016). A Systematic Review of Virtual Reality Simulators for Robot-assisted Surgery. European Urology, 69(6), 1065-1080.

Moore, L. J., Harris, D. J., Sharpe, B. T., Vine, S. J., \& Wilson, M. R. (2019). Perceptualcognitive expertise when refereeing the scrum in rugby union. Journal of Sports Sciences, 37(15), 1778-1786.

Murray, N. P., \& Janelle, C. M. (2003). Anxiety and Performance: A Visual Search Examination of the Processing Efficiency Theory. Journal of Sport and Exercise Psychology, 25(2), 171-187.

Najemnik, J., \& Geisler, W. S. (2005). Optimal eye movement strategies in visual search. Nature, 434(7031), 387-391.

Oberhauser, M., \& Dreyer, D. (2017). A virtual reality flight simulator for human factors engineering. Cognition, Technology \& Work, 19(2), 263-277.

Parsons, T. D. (2015). Virtual Reality for Enhanced Ecological Validity and Experimental Control in the Clinical, Affective and Social Neurosciences. Frontiers in Human Neuroscience, 9. Retrieved January 14, 2020, from https://www.frontiersin.org/articles/10.3389/fnhum.2015.00660/full

R Core Team. (2017). R: A language and environment for statistical computing. $\mathrm{R}$ Foundation for Statistical Computing, Vienna, Austria. URL https://www.Rproject.org/. 
RUNNING TITLE: Visual search in virtual reality

588

589

590

591

592

593

594

595

596

597

598

599

600

601

602

603

604

605

606

607

608

609

610

611

612

613

614

615

616

617

618

Sala, G., \& Gobet, F. (2017). Does Far Transfer Exist? Negative Evidence From Chess, Music, and Working Memory Training. Current Directions in Psychological Science, 26(6), 515-520.

Salar, R., Arici, F., Caliklar, S., \& Yilmaz, R. M. (2020). A Model for Augmented Reality Immersion Experiences of University Students Studying in Science Education. Journal of Science Education and Technology. Retrieved January 9, 2020, from https://doi.org/10.1007/s10956-019-09810-x

Salvucci, D. D., \& Goldberg, J. H. (2000). Identifying fixations and saccades in eye-tracking protocols. Proceedings of the symposium on Eye tracking research \& applicationsETRA '00 (pp. 71-78). Presented at the the symposium, Palm Beach Gardens, Florida, United States: ACM Press. Retrieved February 4, 2019, from http://portal.acm.org/citation.cfm?doid=355017.355028

Saunders, J., Davey, S., Bayerl, P. S., \& Lohrmann, P. (2019). Validating Virtual Reality as an Effective Training Medium in the Security Domain. 2019 IEEE Conference on Virtual Reality and 3D User Interfaces (VR) (pp. 1908-1911). Presented at the 2019 IEEE Conference on Virtual Reality and 3D User Interfaces (VR).

Savelsbergh, G. J. P., Williams, A. M., Kamp, J. V. D., \& Ward, P. (2002). Visual search, anticipation and expertise in soccer goalkeepers. Journal of Sports Sciences, 20(3), 279-287.

Shannon, C. E. (1948). A Mathematical Theory of Communication. Bell System Technical Journal, 27(3), 379-423.

Signorell, A., Aho, K., Anderegg, N., Aragon, T., Arppe, A., Baddeley, A., \& Chessel, D. (2018). DescTools: Tools for Descriptive Statistics. 2015. R package version 0.99, 24. R package version 0.99, 24.

Sireteanu, R., \& Rettenbach, R. (1995). Perceptual learning in visual search: Fast, enduring, but non-specific. Vision Research, 35(14), 2037-2043.

Slater M. (2009). Place illusion and plausibility can lead to realistic behaviour in immersive virtual environments. Philosophical Transactions of the Royal Society B: Biological Sciences, 364(1535), 3549-3557.

Stoet, G. (2010). PsyToolkit: A software package for programming psychological experiments using Linux. Behavior Research Methods, 42(4), 1096-1104. 
RUNNING TITLE: Visual search in virtual reality

619 Stoet, G. (2017). PsyToolkit: A Novel Web-Based Method for Running Online

620

621 Questionnaires and Reaction-Time Experiments. Teaching of Psychology, 44(1), 24-

622 31.

Tabachnick, B. G., \& Fidell, L. S. (1996). Using multivariate statistics. Northridge. Cal.:

623 Harper Collins.

624

Tirp, J., Steingröver, C., Wattie, N., Baker, J., \& Schorer, J. (2015). Virtual realities as

625 optimal learning environments in sport - A transfer study of virtual and real dart

626 throwing. Psychological Test and Assessment Modeling, 57(1), 13.

627

Torralba, A., Oliva, A., Castelhano, M. S., \& Henderson, J. M. (2006). Contextual guidance

628 of eye movements and attention in real-world scenes: The role of global features in

629 object search. Psychological Review, 113(4), 766-786.

630

Trninic, D. (2018). Instruction, repetition, discovery: Restoring the historical educational role 631 of practice. Instructional Science, 46(1), 133-153.

632

Vickers, J. N. (2007). Perception, cognition, and decision training: The quiet eye in action. Human Kinetics.

634

Vine, S. J., Uiga, L., Lavric, A., Moore, L. J., Tsaneva-Atanasova, K., \& Wilson, M. R.

635 (2015). Individual reactions to stress predict performance during a critical aviation

636 incident. Anxiety, Stress, \& Coping, 28(4), 467-477.

637

Wolfe, J. M. (2010). Visual search. Current Biology, 20(8), R346-R349.

638

Wood, G., Batt, J., Appelboam, A., Harris, A., \& Wilson, M. R. (2014). Exploring the Impact 639 of Expertise, Clinical History, and Visual Search on Electrocardiogram Interpretation.

640 Medical Decision Making, 34(1), 75-83.

641

642

643

644

645

646

647 
RUNNING TITLE: Visual search in virtual reality

648

649

650

651

652

653

654

655

656

657

658

659

660

661

662

663

664

665

666

\section{Tables}

Table 1. Demographic information (mean and standard deviation)

\begin{tabular}{r|rrrrr} 
& $\begin{array}{r}\text { Mean } \\
\text { age }\end{array}$ & $\%$ Male & $\begin{array}{r}\text { Previous VR } \\
\text { use }(\%)\end{array}$ & $\begin{array}{r}\text { Video game } \\
\text { experience (max 5) }\end{array}$ & $\begin{array}{r}3 D \text { vision } \\
\text { (max 10) }\end{array}$ \\
\hline & & & & & \\
Training group & $22.4(3.6)$ & 28 & 76 & $3.3(1.5)$ & $5.6(2.6)$ \\
Search group & $23.6(4.7)$ & 44 & 72 & $4.0(1.0)$ & $6.7(1.6)$ \\
Control group & $21.7(2.3)$ & 29 & 73 & $3.9(0.9)$ & $5.9(2.5)$
\end{tabular}

